integration forms are shown, similar in every way to those illustrated in fig. 6 .

FIG. 6. Disintegration forms from lung of rabbit shortly after death. Dry film. Leishman's stain.

The drawings, figs. $3,4,5$, and 6 , were made with a Leit $\frac{1}{16}$ th inch achromatic objective. Leitz Huyghenian ocular No. 5. Draw-tube $170 \mathrm{~mm}$. Magnification approximately 1600 diameters.

\title{
SOME OBSERVATIONS ON THE SUBJECT OF MARGINAL POINTS.
}

\author{
By Walter Jowett, F.R.C.V.S., D.V.H. (Liv.), Department of \\ Agriculture, Cape Town.
}

THE accompanying illustration (fig. I) is a microphotograph of a field in a blood film from one of the experimentally infected cats mentioned in the foregoing article on cattle trypanosomiasis. As reference to the figure will show, a trypanosome is present in the centre of the field, but the object of the present note is more especially to call attention to the coccus-like bodies which are seen in several of the erythrocytes in the same field.

Fig. 2 shows the presence of similar bodies in the red cells. This microphotograph likewise illustrates only one microscopic field, but in this instance the blood film was prepared from a healthy cat, or rather one which had not been inoculated or otherwise experimented with in any way. This particular animal had been purchased locally, and at the time when brought to our notice it was suckling a kitten. Bodies similar in every respect were also present in the erythrocytes of the kitten. Both the mother and her offspring were to all appearance in good health and in fair condition. Some degree of anisocytosis was occasionally observed in the blood films prepared from both these animals, and in the kitten at times normoblasts were seen; otherwise, anæmia or anæmic lesions were not especially evident in either case. Blood counts, unfortunately, were not made in the case of any of the animals mentioned in the present article, stress of other work preventing one from devoting as much time to the study of this condition as one would have liked.

In the centre of the field illustrated in fig. 2 it will be noticed that one of the erythrocytes contains a body which appears to be in process of division, two coccus-like forms lying close together near the edge of the blood cell. Forms similar to these were frequently encountered in all the animals here mentioned.

Coccus-like bodies, identical, as far as one could see, with those figured in the two accompanying illustrations, were found in another apparently healthy cat, as well as in still another, but trypanosomeinfected, subject of the same species.

Most of our trypanosome-infected rats have shown the presence of similar coccus-like bodies in their erythrocytes at one time or another, as also have several of our uninoculated and healthy rats. In all the animals in which we have noted their presence the bodies have persisted for some considerable time. 
In the case of the trypanosome-infected, and consequently anæmic, subjects (both cats and rats) the bodies were, as a rule, more numerously present than in other animals which appeared healthy and which had not formed the subject of experiment. They were sometimes markedly noticeable in the blood cells of our experimental rats the day following the administration of a dose of antimony.

In the trypanosome-infected, and occasionally in the healthy, rats (especially in the young ones) polychromasia and anisocytosis were apparent, the same of course in the cats infected with trypanosomiasis. In these cases the bodies were found distributed equally in the altered and normal erythrocytes.

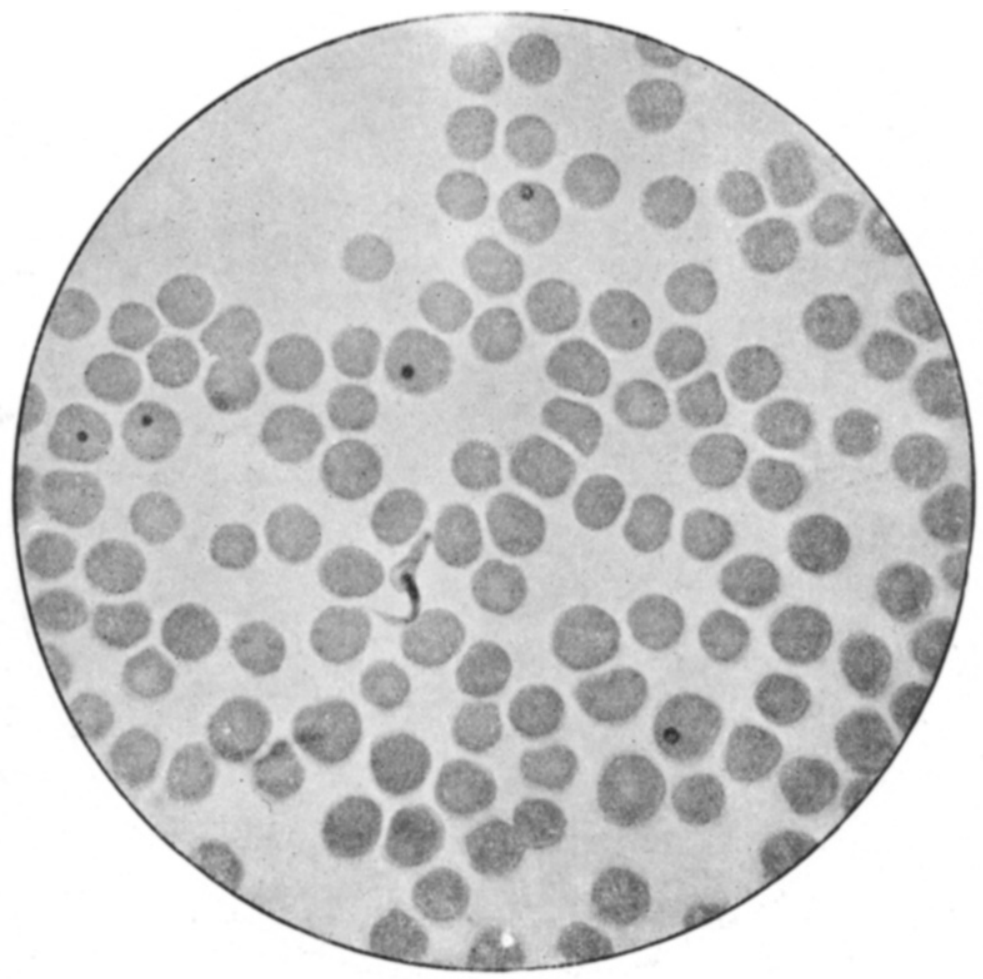

FIG. 1.

The coccus-like bodies here referred to stain well with Romanowsky's method,-a deep red with Leishman's modification. They also stain well with thionin. With Giemsa's stain and azur-eosin they take a purple or deep purple-red to a violet-black tinge. Briefly stated, then, the bodies give the chromatin-staining reaction with the dyes named. It was never possible to distinguish a blue-stained cytoplasm in connection with any of the bodies. In a few instances, however, we observed a pink-stained substance uniting two coccuslike forms which were seemingly in course of division, but which had not yet completely separated from each other. Occasionally a body in an erythrocyte seemed to be surrounded by a clear, unstained, or only faintly stained, halo, whilst sometimes coccus-like bodies were seen free in the blood plasms. 
Intra-corpuscular bodies resembling those here mentioned have been described by other observers who have encountered them in various animals-goats, calves, rats, etc.

Marginal points, coccus-like bodies, or peripheral bodies, are often present in the erythrocytes of cattle in this country, as was first shown by Theiler. They frequently occur in association with certain of the piroplasmata and are sometimes present in considerable numbers. In these animals (cattle) the presence of the bodies is associated with a definite diseased condition, to which, recently, Theiler has given the name anaplasmosis. This investigator has shown that the bodies now referred to (i.e., the marginal points which occur in the red blood

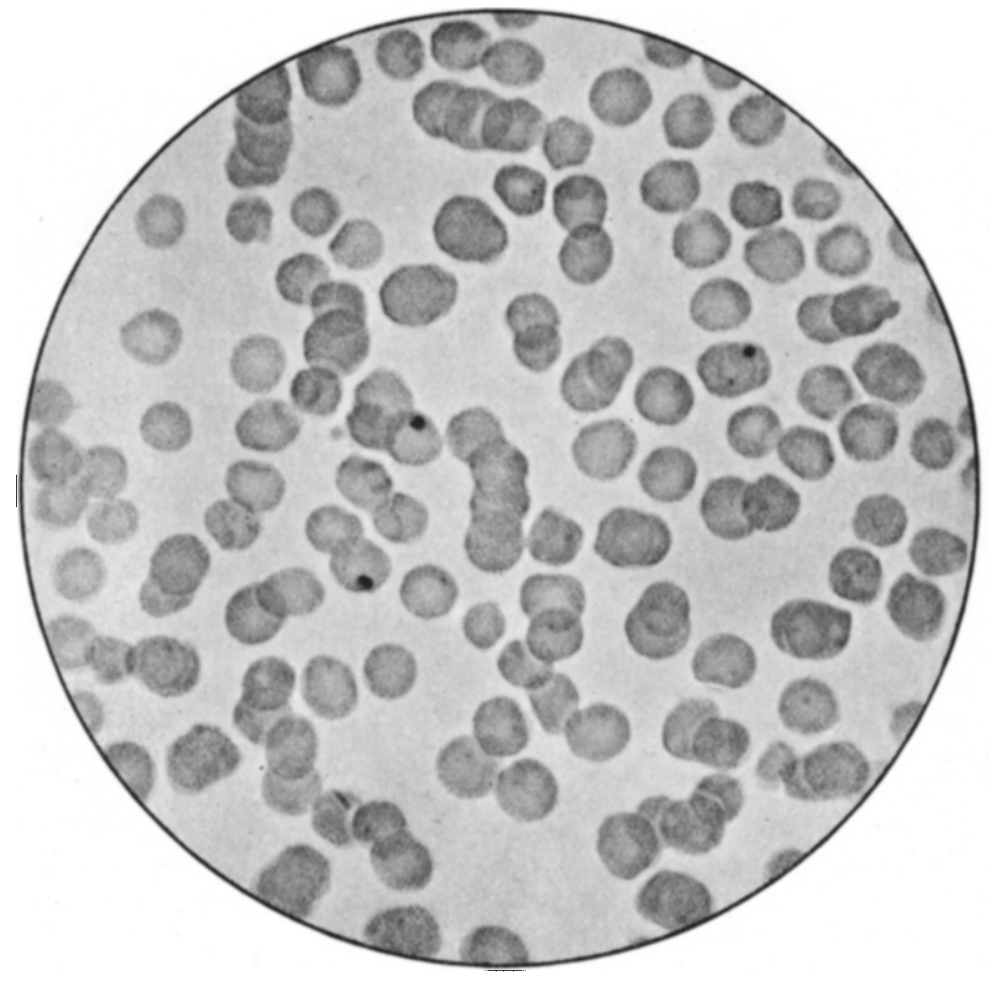

FIG. 2.

cells of cattle) are distinct and definite tick-transmitted protozoan parasites. Theiler considers that they represent a new genus, which he has appropriately designated anaplasma, and to the particular species of this genus met with in cattle he has given the name anaplasma marginale.

The intra-corpuscular coccus-like bodies have apparently a wide geographical distribution. They were first figured and described by Smith and Kilborne in the case of North American cattle, in which they were met with frequently in association with the disease Texas fever, due to the piroplasma (babesia) bigeminum. It was thought, in fact, that they probably constituted a stage in the life cycle of the last mentioned parasite. Knuth has encountered similar bodies in 
the blood of cattle in South America; Lignières the same in the Argentine; and Dschunkowsky and Luhs in Trans-Caucasia. In India Baldrey has seen atypical forms of piroplasmata in the erythrocytes of horses which were suffering from a disease allied to bilious fever; some of the bodies, he considers, resemble the anaplasma marginale of cattle described by Theiler. In some of our own slides, prepared from cases of South African equine piroplasmosis, distinct red-stained coccus-like bodies are present in the erythrocytes.

As a matter of fact, in his original description of the piroplasma equi, Theiler noted the occurrence of these bodies in the red blood cells, as the following extract from one of his articles in the Journal of Comparative Pathology (Vol. XVI., Part 2) will show. He there states: "Upon one occasion I have seen what appeared to be a karysoma persist for a considerable period." Particulars are then given with regard to the case in which these bodies were observed. The subject was an English hackney mare which had contracted the disease under natural conditions, i.e., by means of ticks. Numerous parasites-piroplasma equi-were found present on the 5th March 1902. Under medicinal treatment the mare recovered, the piroplasmata became less numerous, and by the 8th March were very rare. The blood was continuously examined, however, up to the 24th March-for nineteen days after apparent recovery. Regarding the microscopical findings during this period, Dr Theiler observes: "During the whole of the period there was constantly present a round body in a red corpuscle, which, when stained by Laveran's method, was of a deep red colour." This reaction to the stain shows, Theiler considers, that the body is of the nature of a karysoma. No protoplasmic substance could be traced, not even after using different stains. The chromatic body was of the size of the karysoma in the full developed parasite (piroplasma equi); a further peculiarity was that at the beginning it could only be found in the so-called macrocytes or corpuscles of about double the size of an ordinary erythrocyte; and it was not until towards the end of the observation that normalsize blood discs containing these bodies could be observed. More than one body in one corpuscle was never seen. "This fact" says Theiler, "would exclude the possibility of its being simply a basic alteration of the hæmoglobin."

Theiler considered "the chromatic body to be one of the forms in the development of the parasite" (piroplasma equi). He looked upon it, in fact, as probably representing "a latent or dormant form of the piroplasm."

As already mentioned, we have noticed bodies apparently identical with these in some of our blood films from cases of equine piroplasmosis at the Cape. The resemblance shown between the bodies now referred to and those figured and described elsewhere in the present article is certainly very close indeed.

Bruce, Hammerton, Bateman, and Mackie have noted the occurrence of marginal points in cattle (especially calves) in Uganda. These investigators refer to these bodies in the following terms: "If," they say, "these bodies really constitute a new and undescribed parasite, the discovery will be one of great interest. Bodies similar in every way to these are found, however, in healthy young rats, goats, calves, 
etc., so that it is difficult to believe at once in their parasitic nature. Rather," they consider, "would they appear to be cell enclosures due to rapid changes taking place in the blood, such as takes place in young animals or in anæmias. In Amakebe [the name applied by the natives of Uganda to a disease of calves which Bruce and his colleagues have shown to be East Coast fever] they [the marginal points] are sometimes very numerous, and it requires no great stretch of the imagination," these investigators consider, "to see in them the youngest stage of the intra-corpuscular parasite, which, from being round, becomes wedge-shaped, oval or circular, and rod-shaped. It may be," they add, "that both these views are true-that some of the so-called marginal points are remains of chromatin from some previous nuclear structures, and that others are the earliest stage of an intra-corpuscular parasite."

As already stated, the object of the present note is merely to record the occurrence of the coccus-like bodies or marginal points in certain of our laboratory animals. As to their significance, one cannot venture to express a decided opinion in the present instance. It may be that they are parasites, or a stage in the life cycle of some parasite, or, on the other hand, as Bruce and his colleagues have suggested, they may be cell enclosures associated with certain blood changes, or remnants of previous nuclear structures.

In conclusion, one may observe, however, that the fact of the bodies having been seen in the blood corpuscles of apparently healthy animals is not alone a very convincing argument against the supposition that they may be parasites. One is aware, of course, of several protozoan and other parasites which occur in certain animals without fulfilling any, or but a slight, pathogenic rôle therein.

\section{ANAPLASMOSIS IN DONKEYS.}

By Andrew Balfour, M.D., B.Sc., F.R.C.P. Edinburgh, D.P.H. Camb., Director, Wellcome Tropical Research Laboratories, Khartoum.

As is now well known, Theiler ${ }^{1}$ has declared the marginal points so frequently encountered in cases of piroplasmosis in cattle to be anaplasma marginale, a specific protozoan parasite of cattle, and has apparently proved it to be the cause of gall-sickness in South Africa. Spreull ${ }^{2}$ has also declared his conviction that the marginal points are parasitic in nature. If we accept these views, it follows that gallsickness exists in cattle in the Sudan, for I found anaplasma marginale in the blood of cattle suffering from piroplasmosis (zide Third Report of these laboratories, PI. IV., fig. 4.) It was, therefore, with some surprise that, after reading Theiler's article, I remembered having noted that anaplasma marginale was present in large numbers in the blood of several donkeys from Malakal on the White Nile, one of which was suffering from piroplasmosis. I confirmed the observation on re-examining the slide, and noted

1 Theiler, A.: "Gall-Sickness of South Africa. (Anaplasmosis of Cattle)." "Journ. of Comp. Path. and Therap.," Vol. XXIII., Part 2.

2 Spreull, J. : " "Marginal Points,' or a New Intracorpuscular Parasite in the Blood of Cattle in South Africa." Ibid., Vol. XXII., Part 4. 